\title{
Foetal arrhythmias: an enigma of the missed beats
}

\section{Ketki S. Kulkarni*, Purnima R. Satoskar, Vandana Bansal, Rachana Kharate}

Department of Obstetrics and Gynecology, Nowrosjee Wadia Maternity Hospital, Mumbai, Maharashtra, India

Received: 14 November 2018

Accepted: 21 November 2018

\section{*Correspondence:}

Dr. Ketki S. Kulkarni,

E-mail: ketkeekulkarni@yahoo.com

Copyright: (c) the author(s), publisher and licensee Medip Academy. This is an open-access article distributed under the terms of the Creative Commons Attribution Non-Commercial License, which permits unrestricted non-commercial use, distribution, and reproduction in any medium, provided the original work is properly cited.

\section{ABSTRACT}

Background: The synchronised depolarization and repolarisation of the atria and ventricles is achieved by specialised cardiac cells that generate an electrical impulse and propagate it along the conducting system in the myocardial tissue, leading to rhythmic activity of the atria and ventricles. Abnormalities of these rhythms lead to foetal arrhythmia.

Methods: A retrospective observational study was conducted in a tertiary care hospital over period of one year to study the course and perinatal outcome of women with foetal arrhythmias.

Results: The confinement in the study period was $\mathrm{N}=4302$, of which $207(4.81 \%)$ women had foetal congenital malformations. Among them 21(0.48\%) women had foetal cardiovascular abnormalities and $6(0.14 \%)$ women had foetal arrhythmias. There were two cases of maternal primary Sjogrens syndrome: one with foetal 2nddegree atrioventricular (AV) heart block with 2:1 AV conduction and another with foetal 3rd degree or complete heart block. Others were a case of Atrial premature contractions with compensatory pause, a case of Supraventricular tachycardia, one case of sinus tachycardia with non-immune hydrops and a case of foetal bradyarrhythmia with regular ectopic beats in a case of complex congenital heart disease. The course of these pregnancies, treatment options and perinatal outcome was studied.

Conclusions: Foetal echocardiography and Doppler are effective tools for detection and monitoring of foetal arrhythmias. Early and correct diagnoses of arrhythmias help in management of foetal arrhythmias and multidisciplinary team-based approach gives optimum treatment results.

Keywords: AV conduction, Bradyarrhythmia, Complete heart block, Foetal arrhythmia, Second degree block, Sinus tachycardia, Supraventricular tachycardia

\section{INTRODUCTION}

The cardiac cells are specialised and possess a unique property to generate an electrical impulse. The conducting system of the heart propagates the electrical impulse which arises in the sinoatrial (SA) node, through the conducting fibres to the atrioventricular (AV) node. Synchronised depolarization and repolarisation of atrial and ventricular myocardial tissue leads to rhythmic contraction and relaxation of these cavities thereby producing a stable and regular heart rhythm. The AV node acts as a gate and delays the electrical impulse to ventricles allowing atrial contraction followed by ventricular contraction. This delay ensures a coordinated activation of the ventricular myocardium from apex to base. Any abnormalities in these activities can give rise to conduction abnormalities. The conduction system of the foetal heart is functionally mature by 16 weeks of gestation and produces a regular rhythm and rate between 110 and 160 beats per minute (bpm) for the rest of the pregnancy. ${ }^{1}$ The myocytes of the conduction system work in co-ordination and produce contraction, autorhythmicity, intercellular conduction, and electromechanical coupling. Foetal arrhythmia may be 
defined as an irregularity of the cardiac rhythm, as an abnormally slow $(<100 \mathrm{bpm})$ or fast $(>180 \mathrm{bpm})$ heart rate, or as a combination of irregular rhythm and abnormal heart rate. $^{2}$ The types of arrhythmias seen prenatally can be divided broadly into irregular heart rhythms, tachyarrhythmias and bradyarrhythmias.

Irregular heart rhythms mostly occur as 'extra beats' or 'missed beats' and they are due to atrial extrasystoles. An atrial extrasystole occurs and this is followed by a compensatory pause which is perceived as a missed beat on auscultation. Extrasystoles usually occur late in the second or third trimester. In most of the cases, extrasystoles resolve spontaneously prior to delivery, however, frequent bigeminy or frequent trigeminy can cause tachyarrhythmia.

Pathological fetal tachycardias are mostly foetal sinus tachycardia, supraventricular tachycardia, atrial fibrillations, atrial flutter and rarely ventricular tachycardia. In normal circumstances, an electrical impulse is generated in SA node, passes from the atria to the ventricles via the AV node to depolarize the ventricles. Sometimes presence of an accessory pathway can help this electrical impulse to pass rapidly retrograde, from the ventricles to the atria thereby establishing a reentry circuit. In such cases, the time interval between ventricular and atrial contraction is short. Atrial flutter is characterized by a much faster rate of atrial contraction as a result, ventricles cannot respond in a 1:1 contraction and so, the $\mathrm{AV}$ conduction is $\mathrm{A}>\mathrm{V}$. Atrial fibrillation is caused by very fast atrial rates $\sim 400 \mathrm{bpm}$ and completely irregular ventricular rhythm.

Bradyarrhythmias can be sinus bradycardia, and variable $\mathrm{AV}$ conduction blocks. In half of the cases, AV conduction blocks are due to congenital complex heart diseases or in cases where no structural cardiac abnormality is present; they are due to immune mediated damage to the AV node.

With advancing technology, understanding of arrhythmia mechanisms in the foetus is gradually expanding and more and more evolved techniques are being used to the study of foetal rhythm disturbances. Hence a study was done in which foetal arrhythmias were studied in which their diagnosis, as well as their perinatal outcome was studied.

\section{METHODS}

A retrospective observational study of pregnant women with foetal arrhythmias was conducted in a tertiary care hospital over a period of one year from $1^{\text {st }}$ August 2017 to $31^{\text {st }}$ July 2018.

The objectives were to study the perinatal outcome of foetus with arrhythmias; to study the course of foetal arrhythmias and to study the type of foetal conduction abnormalities.

\section{Inclusion criteria}

- Pregnant women with foetal arrhythmia >16 weeks gestation.

\section{Exclusion criteria}

- Pregnant women with foetal arrhythmia whose outcome are not known.

- Pregnant women <16 weeks gestation.

Detailed case studies were done of the women who were diagnosed with foetal arrhythmias in the antenatal period by their antenatal records and indoor case papers. The course of their pregnancy, investigations and reports were analysed and their pregnancy outcome was noted. The foetal outcome and after delivery as well as neonatal outcome were seen. Neonatal course was assessed by neonatal intensive care unit (NICU) notes and daily summaries and treatment sheets. Also, the course of arrhythmia in these infants was assessed by evaluation of their follow up visit records with paediatric cardiologist.

\section{RESULTS}

During the study period of one year, the confinement was 4302. $\mathrm{N}=4302$. Women who delivered or aborted foetus having congenital malformation were 207 (4.81\%). Women with foetus having cardiovascular abnormalities were $21(0.48 \%)$. Amongst them, there were six women with foetal arrhythmias. $(n=0.14 \%)$. The details of each of these cases are as follows:

\section{Case 1: Immune mediated $2^{\text {nd }}$ degree conduction block}

25 years old Primigravida, 29.2 weeks of gestation was referred with foetal bradycardia. Foetal 2D Echocardiography was done and it revealed a structurally normal heart. The ventricular rate was 77 beats/minute and atrial rate of 152 beats /minute. On M Mode echocardiography, there was 2:1 atrioventricular rhythm suggestive of second-degree heart block with $2: 1 \mathrm{AV}$ conduction. There were no signs of foetal heart failure or hydrops. Further investigations revealed anti-nuclear antibodies (ANA) were positive with titres of 1:320 and showing fine speckled pattern. Her ANA BLOT was positive for antibodies against SS-A (soluble substance A) native and Ro. SSA-Ro IgG was positive 166.67RU/ml. Her SSB (soluble substance B)-La IgG was negative $7.40 \mathrm{RU} / \mathrm{ml}$. C3 and C4 Complements were normal.

This patient was managed in a team-based approach with rheumatologist, paediatric cardiologist, and foetal medicine specialist. She was started on oral Dexamethasone $4 \mathrm{mg}$ once daily (OD), Tab. Hydroxychloroquin 300mg OD. She was monitored on fortnightly foetal echocardiography and Colour Doppler. There was no worsening of the atrioventricular block. There were no signs of cardiac failure and hydrops. The 
patient was given an option of elective Caesarean section to avoid strain on foetal heart at the time of labour pains. However, the patient made an informed choice of vaginal trial of labour.

She went in spontaneous labour at 36 weeks and delivered a female with birth weight of $2.9 \mathrm{~kg}$. The APGAR score at birth was- for 1-minute $7 / 10$ and 5minute $9 / 10$. The baby was shifted in neonatal intensive care unit for close observation. The neonate did not require any ventilator support. Post-natal heart rate was 70-76 b/minute.

Respiratory rate was 32/minute. No evidence of cyanosis, no signs of failure. The neonate-maintained oxygen saturation and tolerated breast feeds. Post-natal ECG done on second day showed 2nd degree AV conduction block, Mobitz type I. The neonate was shifted out from the neonatal intensive care unit (NICU) on day 7. Holter monitoring was done and revealed AV block.

The neonate was discharged on day 15. At the end of one month, the ECG showed marked improvement, PR interval was 0.08 seconds and QRS interval was 0.04 seconds. There was also improvement in heart rate on ECHO showing $84-88$ beats/minute. There are no signs of failure and the baby is at present five months old, accepting full feeds and all milestones are well achieved.

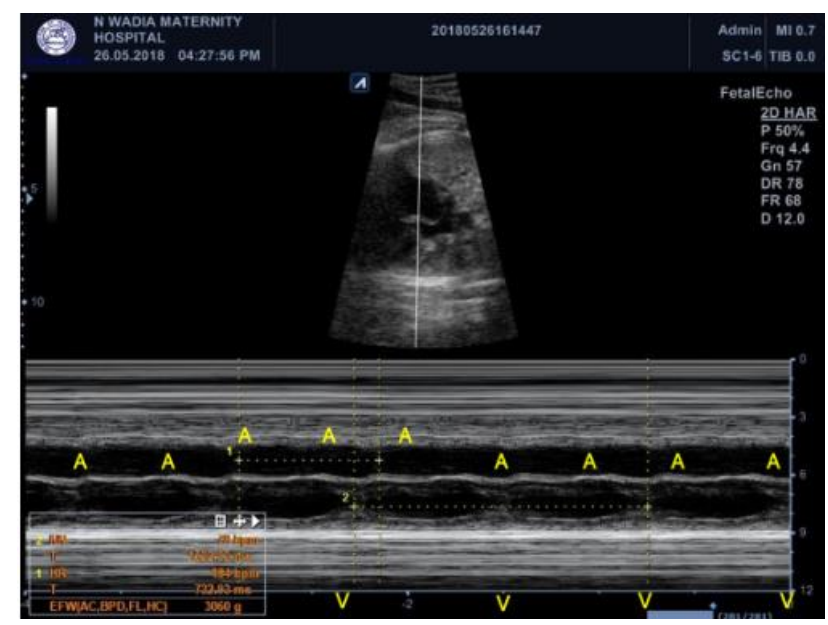

Figure 1: Ultrasound image of $M$ mode foetal echocardiography.

Figure 1 shows ultrasound image of $M$ mode foetal echocardiography. The atrial rate is $164 \mathrm{bpm}$ and the ventricular rate is $78 \mathrm{bpm}$. There are two atrial contractions denoted by 'A' per ventricular contraction denoted by ' $\mathrm{V}$ ' due to $2: 1 \mathrm{AV}$ conduction. Hence, it showed second degree heart block.

Figure 2 shows her intrapartum monitoring trace during labour. Since the foetal ventricular rate was $\sim 70-78 \mathrm{bpm}$, all through her labour, the intrapartum tracing showed severe foetal bradycardia with baseline of $70 \mathrm{bpm}$.

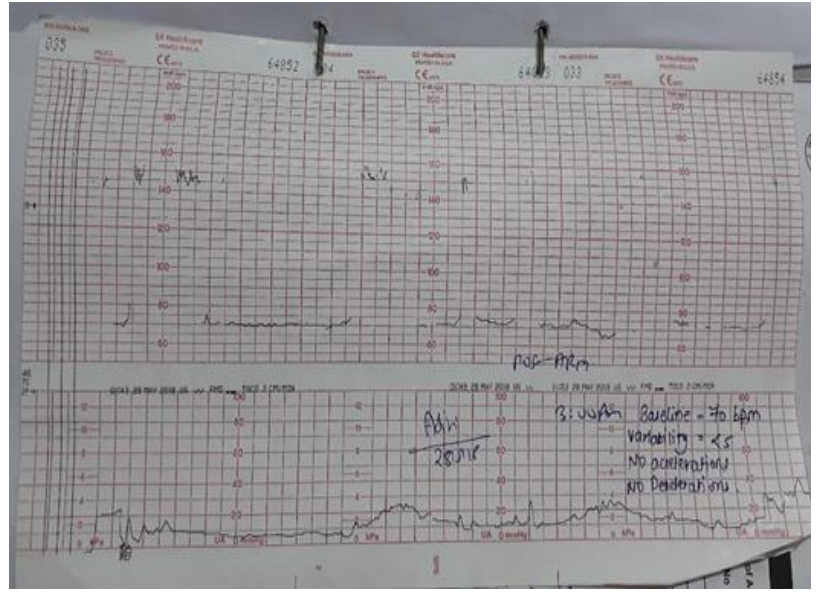

Figure 2: Intrapartum monitoring trace during labour.

Figure 3, the post-natal ECG of the neonate shows second degree AV block: Mobitz I (Wenckebach).

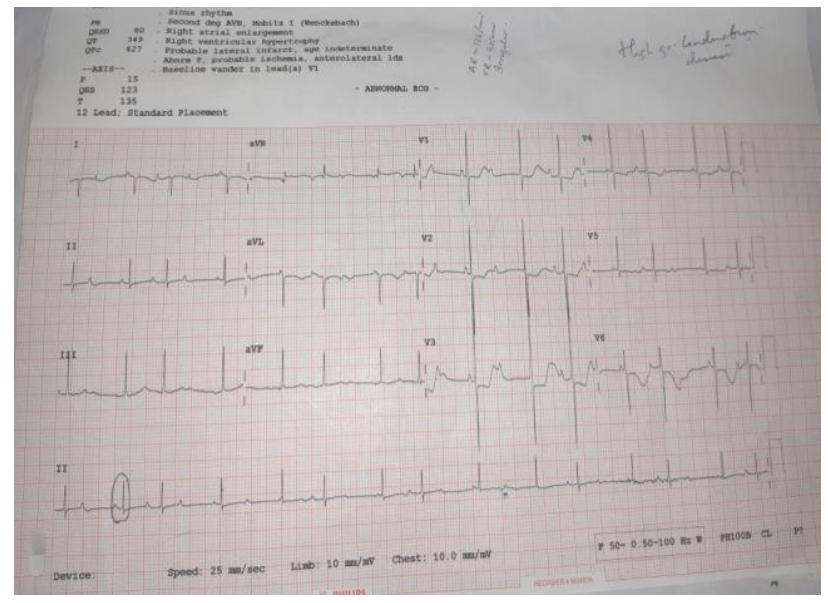

Figure 3: Post-natal ECG of the neonate.

\section{Case 2: Immune mediated complete heart block}

30 years, G3P2L1IUFD1 26 weeks of gestation came in antenatal clinic. Her first pregnancy was a 29 weeks intrauterine foetal demise. There was a history of peripartum cardiomyopathy in that pregnancy. Second pregnancy was an elective Caesarean section for breech presentation. Present pregnancy was a spontaneous conception. On further evaluation, foetal 2D ECHO was done which revealed complete heart block with a structurally normal foetal heart and a ventricular heart rate of 62 beats/min. Maternal investigations revealed that she was Anti -Ro, anti-La Antibodies positive. She was started on Tab. Dexamethasone $4 \mathrm{mg}$ and monitored. She eventually developed deranged blood sugars in her antenatal period and had to be started on tablet Metformin. The foetus was monitored for hydrops and cardiac failure by fortnightly foetal echocardiography. The patient started developing intermittent breathlessness at around 32 weeks of gestation. 
Subsequent ultrasonography revealed foetal growth restriction but no signs of cardiac failure. She was managed and monitored closely with cardiologist and at 36 weeks of gestation, elective caesarean section was done for worsening breathlessness. A male baby of 1.904 $\mathrm{kg}$ was delivered. It was an intrauterine growth restricted baby with heart rate of $62 \mathrm{~b} / \mathrm{min}$. Post-natal ECG showed complete heart block with ' $p$ ' and 'qrs' complexes completely independent of each other. The neonate did not require any ventilator support, maintained oxygen saturation and was tolerated full feeds in which were given in form of expressed breast milk. The baby was evaluated by paediatric cardiologist and decision taken of pacemaker if the baby starts to develop signs of failure. The infant at the end of four weeks also did not require pacemaker and showed no signs of failure and tolerating full feeds.

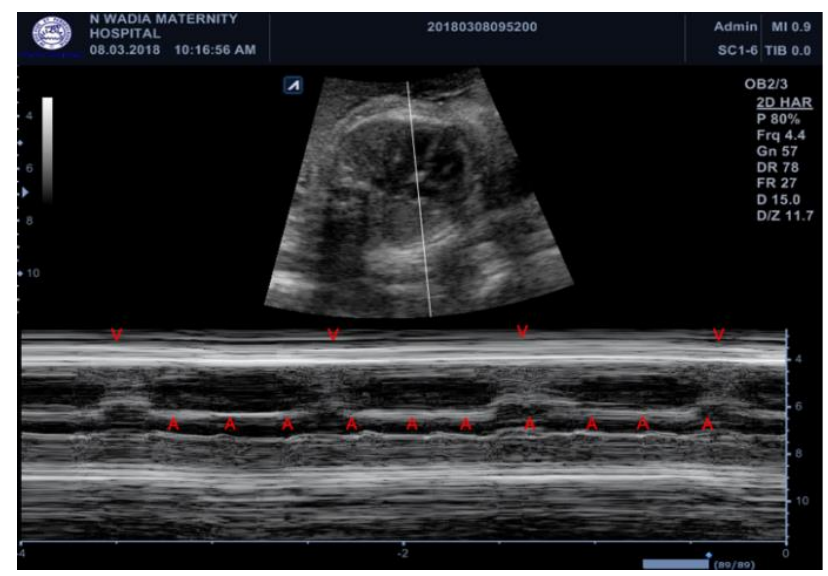

Figure 4: Foetal M mode echocardiography.

Figure 4 shows foetal $M$ mode echocardiography in which ventricles denoted by ' $\mathrm{V}$ ' and atrial activity denoted by ' $A$ ' is completely dissociated showing complete heart block.

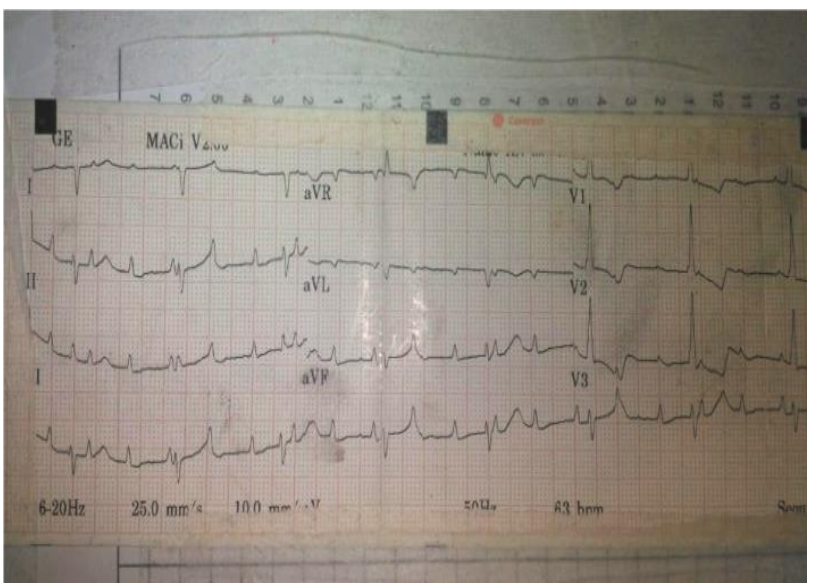

Figure 5: Post-natal ECG of the neonate done on day 1 of life.

Figure 5 shows post-natal ECG of the neonate done on day 1 of life in which the ' $p$ ' wave and 'qrs' complexes are occurring independent of each other, suggesting third degree or complete heart block.

\section{Case 3: Bradyarrhythmia in a case of complex congenital heart disease}

G2A1 aged 20 years, 19.4 weeks of gestation, was detected to have foetal bradycardia in the antenatal outpatient department. Her level III ultrasound for foetal malformation revealed corrected transposition of great arteries, a large subpulmonic ventricular septal defect (VSD) with right to left shunt and L posed aorta. Also, foetal bradycardia 55b/min with regular ectopics were seen. This patient eventually opted for medical termination of pregnancy (MTP) and second trimester MTP was done.

\section{Case 4: Supraventricular tachycardia}

19 years old primigravida, 33.3 weeks of gestation, came in the emergency department with severe foetal tachycardia: foetal heart rate was $220 \mathrm{bpm}$. Her level III antenatal scan done at 20.5 weeks of gestation was normal. Maternal pulse was normal. There was a history of threatened preterm labour a day prior for which tocolysis in form of injectable isoxsuprine was given by the treating doctor. Maternal s.TSH was normal. Maternal ECG was normal. Foetal 2D ECHO showed foetal tachycardia: heart rate $\sim 250 \mathrm{~b} / \mathrm{min}$ with no irregularity and 1:1 AV conduction. It also showed minimal pleural effusion and minimal pericardial effusion. Hence a diagnosis of supraventricular tachycardia (SVT) with early signs of cardiac failure was made. She was given T.Digoxin $0.5 \mathrm{mg}$ loading dose and repeat dose of 0.25 mg was given after $8 \mathrm{hrs}$. Meanwhile two doses Injection Betamethasone was given. There was no response and the supraventricular tachycardia did not subside. Hence emergency caesarean section was done, and a female $2.420 \mathrm{~kg}$ was delivered with APGAR-1 min- 8/10, 5 min9/10. There was spontaneous correction of SVT after delivery and foetal heart rate $\sim 140 \mathrm{~b} / \mathrm{min}$. The neonate was shifted to NICU for observation and monitoring. In NICU, the neonate developed repeat episode of SVT after 3 days of birth for which inj adenosine $100 \mu / \mathrm{kg} / \mathrm{dose}$ was given IV push to abort the tachyarrhythmia. The baby had repeated episodes of SVT hence the baby was started on oral Propranolol and oral Flecainide. Repeat neonatal ECHO was normal. ECG showed QRS interval $0.08 \mathrm{sec}$ and PR was $0.16 \mathrm{sec}$ and multiple premature complexes, ventricular as well as supraventricular with signs of left atrial enlargement. Karyotyping was done which revealed normal karyotype. However, the baby had few more episodes of SVT and hence T. Amiodarone was added.

An ambulatory Holter recording was done which revealed occasional atrial ectopics and few episodes of sustained atrial tachycardia longest episode lasting 23 minutes with heart rate around 270 beats/min. The infant is on tablet flecainide and tablet amiodarone and at present ten months old. All milestones are well achieved. 


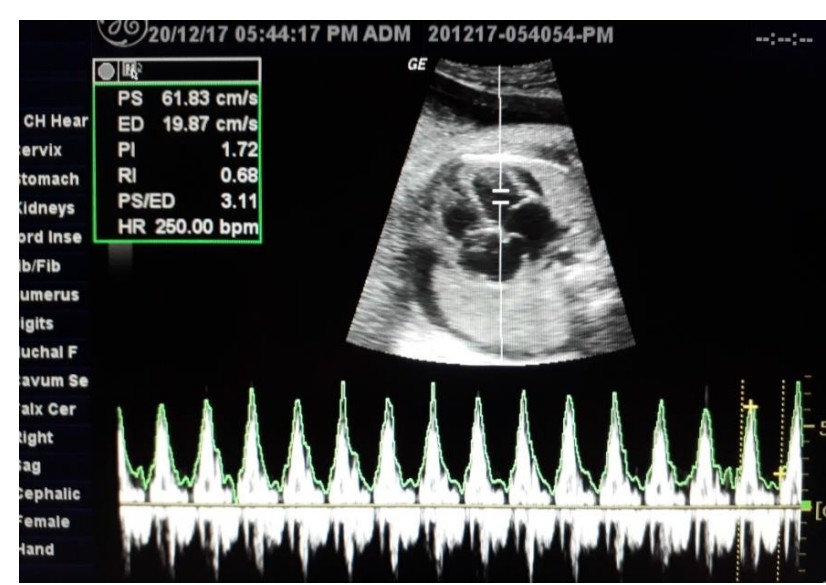

Figure 6: Ultrasound image of supraventricular tachycardia.

Figure 6 shows ultrasound image of supraventricular tachycardia-the heart rate was $250 \mathrm{bpm}$. A thin rim of pericardial effusion can also be seen.

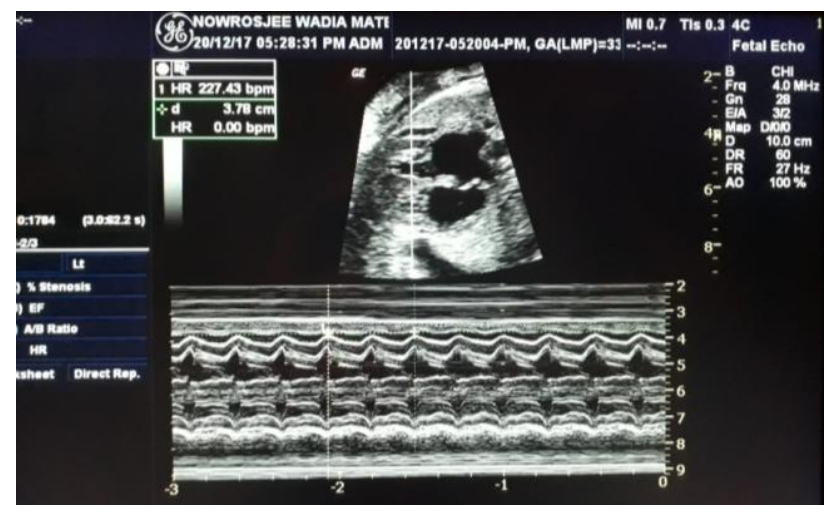

Figure 7: M mode foetal echocardiography.

Figure 7 shows $M$ mode foetal echocardiography in which there is severe foetal tachyarrhythmia with 1:1 AV conduction, hence supraventricular tachycardia.

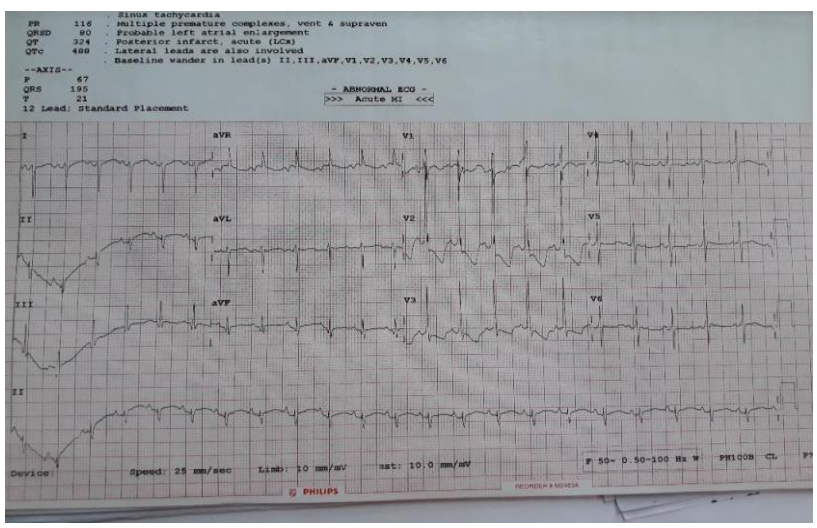

Figure 8: Neonate's post-natal ECG.

Figure 8 shows post-natal ECG of the neonate which shows multiple premature complexes, ventricular and supraventricular.

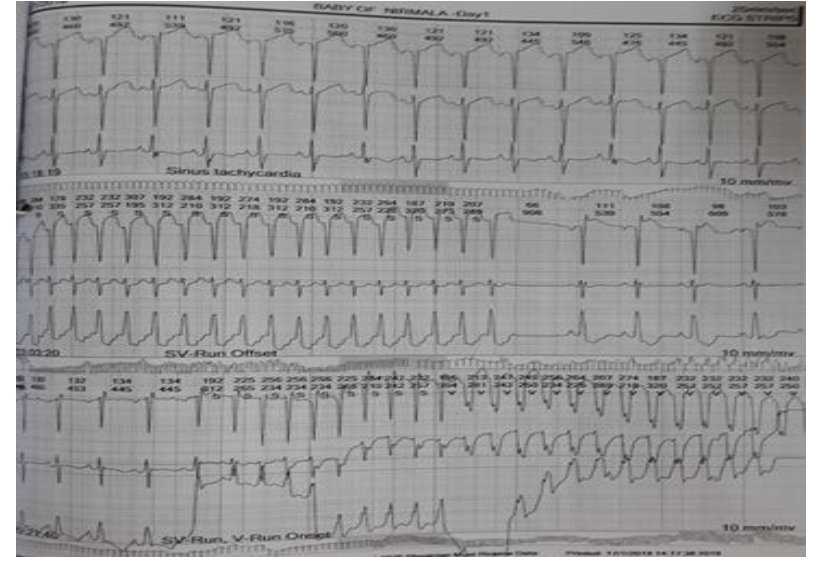

Figure 9: Holter monitoring of the neonate.

Figure 9 shows the Holter monitoring of the neonate which shows intermittent runs of supraventricular tachycardia.

\section{Case 5: Premature atrial ectopics with compensatory pause.}

A 27 years old primigravida, known case of gestational diabetes mellitus came with ultrasonography in third trimester suggestive of borderline foetal tachycardia. Maternal serum TSH-normal. Her level III ultrasound for foetal malformation was done at 19 weeks and was normal. Foetal 2D ECHO showed few intermittent premature atrial contractions followed by compensatory pause. And foetal heart rate $\sim 150-160$ beats/minutes with a structurally normal heart. Maternal ECG was normal. No active management was done as the ectopic beats were few and intermittent. Glycaemic control was ensured, and calcium supplementation was given. On subsequent foetal 2D ECHO, premature atrial ectopics were not observed. The patient delivered a male baby, $2.945 \mathrm{~kg}$ at 39.3 weeks of gestation by emergency caesarean section. Neonatal heart rate was 142 beats/minute, and no premature beats. APGAR score was at one minute- 9/10. Post-natal 2D ECHO was normal. Baby was discharged on fifth post-operative day.

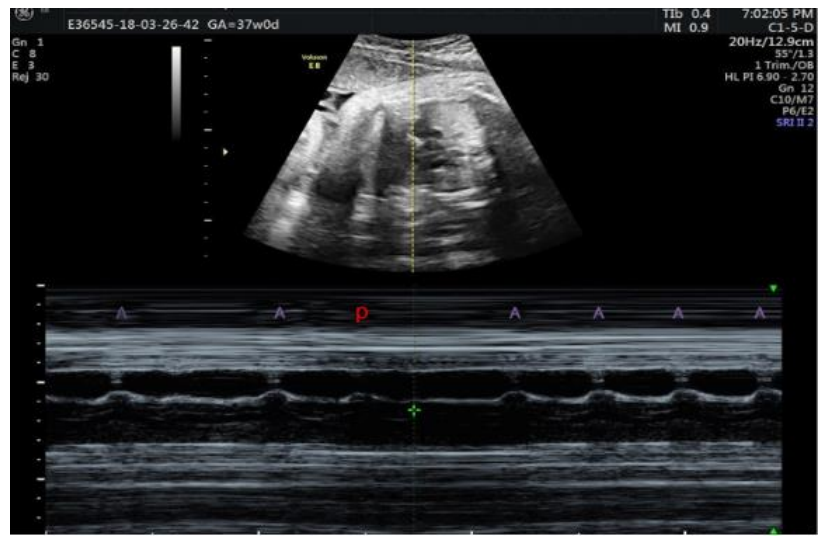

Figure 10: Atrial premature contraction. 
Figure 10 shows atrial premature contraction denoted by 'P' with a compensatory pause.

\section{Case 6: Sinus tachycardia in a case of non-immune hydrops}

G3P2L2 with 29 weeks of gestation with non-immune hydrops was detected to have sinus tachycardia. Foetal heart rate was persistently 180 beats per minute. On foetal 2D ECHO, the heart was structurally and functionally normal except for severe tachycardia with 1:1 AV conduction. Hence a diagnosis of sinus tachycardia was made in a foetus with non-immune hydrops. Maternal pulse was normal. Maternal s. TSH, ECG, TORCH titres and haemoglobin electrophoresis were normal. The parents were not willing for invasive prenatal testing for diagnosis of non-immune hydrops.

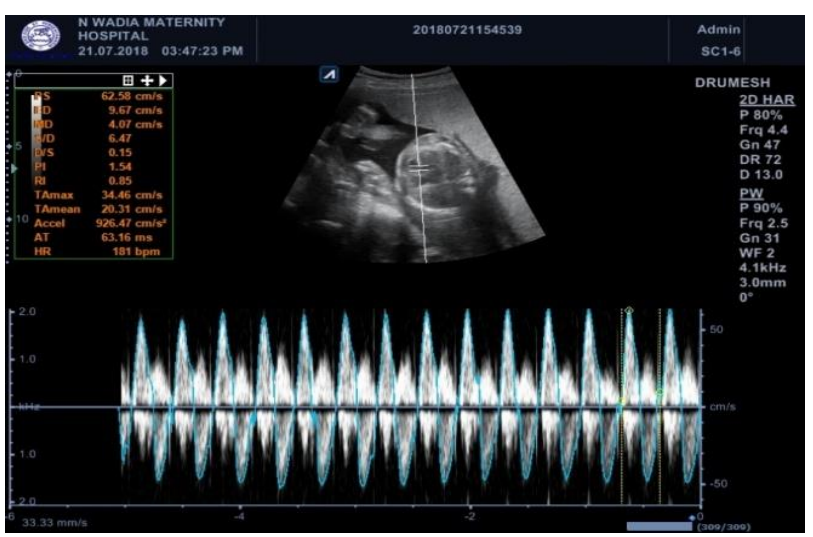

Figure 11: Foetal sinus tachycardia.

Hence cause of non-immune hydrops was found could not be ascertained. At 29 weeks of gestation, she started developing preeclampsia, slowly progressed to severe preeclampsia and eventually labour was induced at 31 weeks in view of severe preeclampsia with impending eclampsia. She delivered a $1.89 \mathrm{~kg}$ male baby with APGAR score of $4 / 10$ at one minute and $5 / 10$ at five minutes and heart rate of 170 beats / minute. The neonate expired within 3 hours of birth due to multiorgan failure with non-immune hydrops.

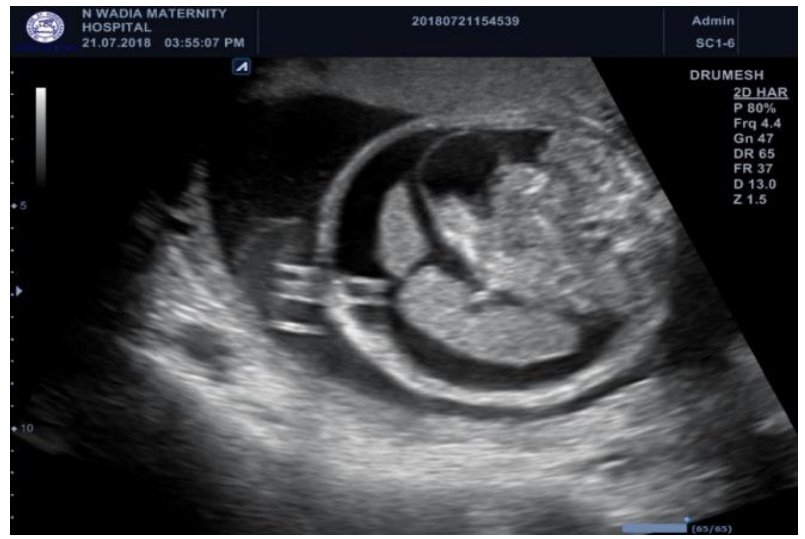

Figure 12: Foetal non-immune hydrops.
Figure 11 shows foetal sinus tachycardia (foetal heart rate persistently $\sim 180 \mathrm{bpm}$.). The foetal heart was structurally normal, but the foetus had non-immune hydrops.

Figure 12 shows foetal non-immune hydrops.

\section{DISCUSSION}

The incidence of women with foetal arrhythmias in present study was 6 cases in 4302 women which is $0.14 \%$. This is lower than the incidence of 1 to $2 \%$ quoted in literature. ${ }^{2}$ All the cases of foetal arrhythmias could be picked up by $2 \mathrm{D}$ ultrasonography with $\mathrm{M}$ mode ECHO and Colour and pulsed Doppler. These modalities are effective to study the rate and timing of atrial and ventricular mechanical events which occur briefly after their respective electrical depolarization. ${ }^{3,4}$ Echocardiography also has an important role in follow-up of foetus with arrhythmias. The assessment of complications of arrhythmia can be performed such as evaluation of deterioration of myocardial function, valvular regurgitation and development of hydrops., 5 Doppler evaluation is also used for the evaluation of 'mechanical PR intervals' as a surrogate for 'PR' interval measured on ECG or MCG. ${ }^{7-9}$ The limitations of using magnetocardiograms is limited availability of the technique and the need for a magnetically shielded room for the study. ${ }^{10-12}$

In present study too, all the arrhythmias were diagnosed on ultrasonography using M mode echocardiography and colour and pulsed Doppler. Irregular heart rhythms can be detected during routine auscultation of the foetal heart or routine ultrasound. They are due to atrial extrasystoles. ${ }^{13}$ Extrasystoles occur late in the second or third trimester and are associated with a $2-3 \%$ risk of tachyarrhythmias or other co morbidity but in most cases, these arrhythmias resolve spontaneously prior to delivery. ${ }^{13}$

In present study too, the intermittent atrial ectopic did not require any specific intervention. Tachyarrhythmias are defined as foetal heart rates of more than 180 beats per minute ${ }^{14}$. These are broadly classified as sinus tachycardia, SVT, atrial flutter and ventricular tachycardia. Persistent sinus tachycardia has 1:1 AV conduction and they occur in conditions such as foetal anaemia, foetal hypoxia, infections and thyrotoxicosis. Atrial flutter is caused by a fast rotating macro-reentrant circuit that is confined to the atrium. ${ }^{2}$ So the atrial rate is very high around 300 and $500 \mathrm{bpm}$ due to which only every second or third atrial beat is conducted across the AV node. ${ }^{2}$ This results in ventricular rates between 150 and $250 \mathrm{bpm}^{2}$ and hence $\mathrm{A}: \mathrm{V}$ conduction ratio $1>1$. Supraventricular tachycardia (SVT) is intermittent and occurs due to AV re-entry through a fast conducting accessory pathway. Since the retrograde pathway conduction is faster, the atrium is excited immediately after the ventricle, which causes a heart rate of 180 and $300 \mathrm{bpm}^{2}$. However, conduction and contractility of atria 
and ventricles show a 1:1 AV relationship. ${ }^{2}$ The supraventricular tachycardia can have sudden onset and abrupt termination of tachycardia.

In present study, antiarrhythmic drug in form of maternal treatment with digoxin was given as there were signs of cardiac decompensation in form of pericardial effusion. However, there was no response to digoxin in present case. Authors also observed that delivery itself caused abrupt and sudden conversion of the tachyarrhythmia into sinus rhythm.

Bradyarrhythmias - Fetal bradycardia is conventionally defined by a decrease in heart rate $<100 \mathrm{bpm}^{2}$. These can be caused by persistent sinus bradycardia, blocked atrial bigeminy, atrioventricular conduction blocks, left atrial isomerism, severe foetal distress, chronic and severe hypoxia. Immune mediated blocks occur due to variable damage to the AV node by passively acquired autoimmune disease in which maternal autoantibodies to the intracellular ribonucleoproteins Ro (SS-A) and La (SS-B), cross the placenta and injure the previously normal fetal heart. ${ }^{2}$ These antibodies trigger the inflammation of the AV node and the myocardium which heal with fibrosis and may cause heart block. ${ }^{2}$

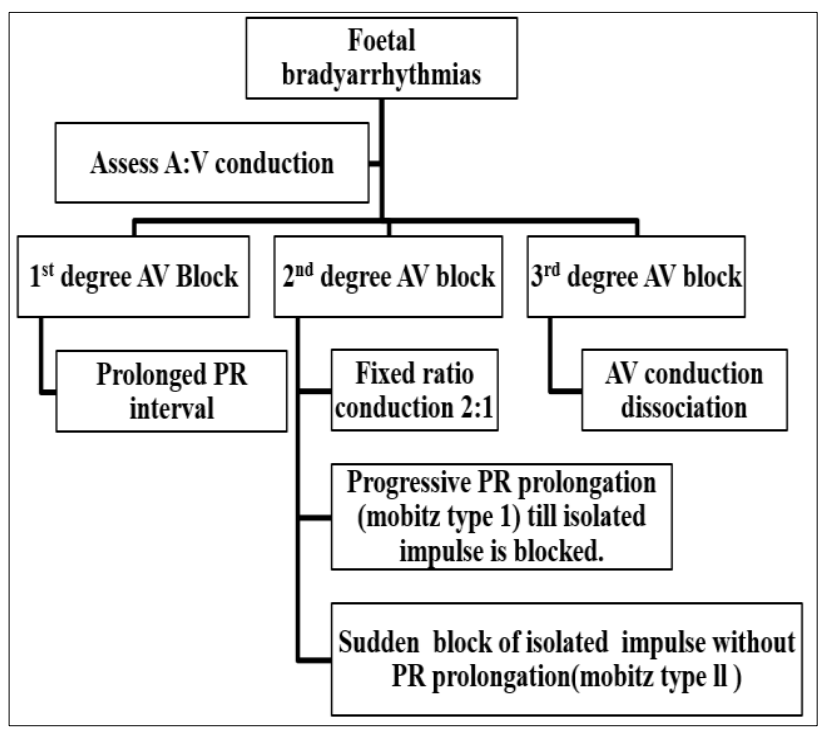

Figure 13: Algorithm for the evaluation of bradyarrhythmias with AV conduction defects.

Figure 13 shows algorithm for the evaluation of bradyarrhythmias with AV conduction defects.

Many studies in literature have shown that steroids, especially fluorinated steroids are first line treatment in the treatment of immune mediated blocks. In present study too both the patients with immune mediated AV conduction blocks were treated with dexamethasone, a fluorinated steroid. In both cases, it did not reverse the heart block; however, it prevented worsening of the block, prevented immune mediated myositis which causes hydrops and heart failure.

\section{CONCLUSION}

The management of foetal arrhythmias require multidisciplinary team-based approach consisting of an obstetrician, foetal medicine specialist, paediatric cardiologist, rheumatologist, and neonatologist. Ultrasonography using $\mathrm{M}$ mode and Doppler and foetal 2D ECHO are effective tools in diagnosing, monitoring and surveillance of foetal arrhythmias. Correct diagnosis of type of arrhythmias is essential as different types of arrhythmias require different treatment.

Transient arrhythmias in form of atrial premature beats in absence of structural heart disease require no active treatment. Supraventricular tachycardia can be treated by maternal treatment with digoxin if foetus shows signs of cardiac decompensation. However, delivery can also cause spontaneous correction of supraventricular tachycardia. Fluorinated steroids are treatment of choice in cases of immune mediated AV conduction blocks. Steroids did not reverse the foetal arrhythmia in AV conduction blocks but prevented myositis and progression to heart failure.

Funding: No funding sources

Conflict of interest: None declared

Ethical approval: Not required

\section{REFERENCES}

1. Macones GA, Hankins GD, Spong CY, Hauth J, Moore T. The 2008 National Institute of Child Health and Human Development workshop report on electronic fetal monitoring: Update on definitions, interpretation, and research guidelines. Obstet Gynecol. 2008;112(3):661-6.

2. Weber R, Stambach D, Jaeggi E. Diagnosis and management of common fetal arrhythmias. J Saudi Heart Assoc. 2011;23(2):61-6.

3. Jaeggi ET, Fouron JC, Fournier A. Ventriculo-atrial time interval measured on M-mode echocardiography: a determining element in the diagnosis, treatment and prognosis of fetal supraventricular tachycardia. Heart. 1998;79:582-7.

4. Fouron JC, Proulx F, Miro J. Doppler and M-mode ultrasonography to time fetal atrial and ventricular contractions. Obstet Gynecol. 2000;96:732-6.

5. Hofstaetter C, Hofstaetter C, Hansmann M, Eik-Nes SH, Huhta JC, Luther SL. A cardiovascular profile score in the surveillance of fetal hydrops. J Matern Fetal Neonatal Med. 2006;19:407-13.

6. Huhta JC. Right ventricular function in the human fetus. J Perinat Med. 2001;29:381-9.

7. Rosenthal D, Friedman DM, Buyon J, Dubin A. Validation of the Doppler PR interval in the fetus. J Am Soc Echocardiogr. 2002;15:1029-1030.

8. Bergman G, Jacobsson LA, Wahren-Herlenius M, Sonesson SE. Doppler echocardiographic and electrocardiographic atrioventricular time intervals in newborn infants: evaluation of techniques for 
surveillance of fetuses at risk for congenital heart block. Ultrasound Obstet Gynecol. 2006;28:57-62.

9. Friedman DM, Kim MY, Copel JA, Davis C, Phoon CK, Glickstein JS, et al. Utility of cardiac monitoring in fetuses at risk for congenital heart block: the PR Interval and Dexamethasone Evaluation (PRIDE) prospective study. Circulation. 2008;117:485-93.

10. Horigome H, Ogata K, Kandori A, Miyashita T, Takahashi-Igari M, Chen YJ, et al.Standardization of the PQRST waveform and analysis of arrhythmias in the fetus using vector magnetocardiography. Pediatr Res. 2006;59(1):121-5.

11. Zhao H, Cuneo BF, Strasburger JF, Huhta JC, Gotteiner NL, Wakai RT. Electrophysiological characteristics of fetal atrioventricular block. J Am Coll Cardiol. 2008;51:77-84.
12. Hornberger LK, Collins K. New insights into fetal atrioventricular block using fetal magnetocardiography. J Am Coll Cardiol. 2008;51:85-6.

13. Simpson J, Yates RW, Sharland GK. Irregular heart rate in the fetus- not always benign. Cardiol Young. 1996;6:28-31.

14. Strasburger JF. Prenatal diagnosis of fetal arrhythmias. Clin Perinatol. 2005;32:891-912.

Cite this article as: Kulkarni KS, Satoskar PR,

Bansal V, Kharate R. Foetal arrhythmias: an enigma of the missed beats. Int J Reprod Contracept Obstet Gynecol 2018;7:4862-9. 